ש) SOCIAL AND ETHICAL ISSUES IN 2021

\title{
Staying on track to achieve racial justice in kidney care
}

\section{Dinushika Mohottige and Keisha Gibson (1)}

The dramatic increase in advocacy and scholarly work on the impact of structural racism on health inequities that began in 2020 has been sustained in the past year. In response to the call for action on these issues, the nephrology community has developed policy-based mitigation strategies and continues to examine our role in promoting health equity and justice in the care of patients with kidney disease.

A national reckoning with racism in the United States, amplified by persistent racial and ethnic disparities in access to COVID-19 vaccines and in COVID-19-related morbidity and mortality, has galvanized kidney care communities to sustain attention to what Bignall and Crews described as an imperative to commit to social justice and equitable outcomes ${ }^{1}$. In the past year, rigorous discussions have continued regarding policy and other interventions required to disrupt kidney health inequalities, which include a persistent two- to fourfold higher incidence of kidney failure among Black versus white individuals, a disproportionate representation of Black people on dialysis, the persistently lower access of Black people to optimal therapies including kidney transplantation and SGLT2 inhibitors, and disparities in pre-dialysis nephrology care ${ }^{2-4}$. While glaring disparities in access to care exist worldwide, several articles published in 2021 showcased the need for a collective, well-funded and sustained commitment to justice and equity to undo the devastating impact of structural racism and other forms of bias and inequity on kidney and other health outcomes in the United States.

A key step in dismantling structural racism involves the recognition of racism and race essentialism (that is, the belief that races are biologically distinct groups) as a threat to rigorous science and health equity. In medicine, these beliefs have been perpetuated and their harms propagated by the inclusion of race in clinical algorithms. The nephrology community is, to our knowledge, the first medical subspecialty to interrogate the inclusion of race in a clinical algorithm. Substantial tensions regarding the harms of racialized medical practices, including the long-standing inclusion of a Black race modifier in equations to estimate kidney function, prompted a comprehensive review of this practice by the American Society of Nephrology and the National Kidney Foundation in 2021, with input from multidisciplinary stakeholders including patients and their families. Race modifiers were initially included in kidney function estimation equations to account for observed differences in serum creatinine levels measured in non-generalizable cohorts of Black and white study participants in the United States, despite scientifically invalid explanations regarding the causes of these differences (for example, differences in muscle mass between groups). The harms of this race-based medical practice were found to include a lower detection of chronic kidney disease (CKD) among individuals racialized as Black with a disproportionate burden of rapidly progressive CKD and kidney failure, lower provision of interventions for CKD management, late nephrology referral, and slower access to kidney transplant waiting lists by a median of 1.9 years ${ }^{5,6}$. After several months of systematic deliberation, including the rigorous assessment of 26 approaches for estimating glomerular filtration rate, the taskforce provided unequivocal guidance that CKD Epidemiology Collaboration (CKD-EPI) equations based on serum
Several articles ... showcased the need for a collective, well-funded and sustained commitment to justice and equity

creatinine levels should be implemented across all US laboratories without a race modifier ${ }^{7}$. The taskforce also recommended that access to cystatin $\mathrm{C}$ measurements should be expanded for the purpose of confirming kidney function estimates among individuals at risk of CKD. These recommendations bolster support for investigations that aim to reduce racial and ethnic disparities in kidney disease and advocate for the availability of appropriate tools with which to estimate kidney function using novel biomarkers that are not impacted by social variables, such as race.

Racism must be named and addressed as a root cause of racial disparities in kidney health, including access to transplantation. As described by Purnell et al., racism operates at an individual, interpersonal and structural level to generate a cascade of barriers to kidney transplantation ${ }^{8}$. Racism affects many stages of the pathway to kidney transplantation, including primary care and kidney disease surveillance, referral to nephrology care, the process of transplantation referral, and transplantation evaluation and listing. Factors that arise as a consequence

\section{Key advances \\ - The nephrology community is leading by example to address harms of racialized medical practices in clinical algorithms by removing the long-standing inclusion of a Black race modifier in equations to estimate kidney function in patients? \\ - Efforts to ensure equitable access to transplantation and equitable outcomes for all transplant patients will remain unrealized unless we acknowledge and counter systemic, institutional and interpersonal racism ${ }^{6,8}$. \\ - Associations between racial disparities in the built environment, such as the integrity of community water sources, can be linked with racial disparities between Black and white patients diagnosed with kidney failure ${ }^{9}$.}


Racism must be named and addressed as a root cause of racial disparities in kidney health

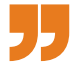

of structural racism, including disparities in access to health care and insurance, wealth inequities perpetuated by unequal hiring and discriminatory practices, persistent resource deprivation and disinvestment in racially segregated neighbourhoods (the result of historic redlining in the United States), and unequal access to culturally and literacy-tailored educational materials to aid treatment decisions, create environments in which minoritized individuals experience structural barriers to kidney care. These barriers include the capacity to fulfil key criteria required for transplantation evaluation, such as the completion of pre-transplantation testing, financial support, and sufficient mechanisms to ensure treatment adherence. Interpersonal racism and other forms of bias manifesting as suboptimal education, communication, and shared or informed decision-making disproportionately affect Black and other minoritized individuals in kidney care, including during the transplantation evaluation process. Potential solutions to these multifactorial problems include strengthening structural competency among providers and health-care organizations. The impact of socio-political factors must be addressed, including the effects of structural racism on clinical care and other health drivers such as transportation access, health literacy and neighbourhoodlevel resources, including clean water. A study from 2021 highlighted the impact of the ageing US water system, reporting higher rates of lead exposure and subsequent lower haemoglobin concentrations and higher use of erythrocyte-stimulating agents among Black versus white patients with advanced kidney disease? ${ }^{9}$.

This renewed lens on dismantling disparities re-contextualizes barriers to access, including 'non-adherence', which is often a label applied to patients who are hindered from meeting the expectations of their health-care team due to the consequences of inequitable systems. Instead, this lens recognizes the critical role of remedying the structural inequalities, such as unequal access to transportation, high-quality food and high-quality mental health care, that disproportionately burden minoritized communities. More efforts are needed to embed anti-racist, anti-biased practice and education into key elements of clinical practice (for example, the training of transplantation selection committees), so that biased and subjective perceptions do not implicitly or explicitly impede care. The implementation of such approaches, coupled with coordinated interventions that earn trust, enhance community capacity (via community-engaged education and advocacy) and enhance multi-stakeholder engagement (with patient navigators and other key individuals) are needed to achieve equity in kidney care outcomes.

It has been reiterated by leading scientific organizations, including the US National Institutes of Health, that race is a sociopolitical variable and has no inherent genetic or biologic meaning. Genetics should therefore not be used to reinforce concepts of racial purity or the fallacy that race captures biologically or genetically distinct and defined groups. By contrast, evidence abounds that the experience of being a minoritized person in a society in which racial stratification occurs, including the United States, is a risk factor for racism and thus for poor or disparate health outcomes. Further investigations are needed to accurately measure structural racism and explore how racism influences biology via weathering, which speaks to the sustained toll of experienced discrimination via allostatic load, epigenetic modifications and other factors. The nephrology community has a unique opportunity to lead by example in dismantling decades of faulty race-based pedagogy. To achieve this goal, we must enhance publication standards to ensure that race is appropriately contextualized and defined in health studies; enhance multidisciplinary efforts to understand and address racial kidney health disparities, with input from geneticists, social scientists and other disciplinary experts; and embed a race-conscious, equity-oriented approach to the design, conduct and analyses of research. By taking these steps, the nephrology community will not only remain on track but successfully reach our goal of achieving health equity and justice for patients with kidney disease.

Dinushika Mohottige ${ }^{\prime}$ and Keisha Gibson (D) ${ }^{2 \times}$ 'Division of Nephrology and Hypertension, Duke University Medical Center, Durham, NC, USA.

${ }^{2}$ UNC Kidney Center, University of North Carolina at Chapel Hill, Chapel Hill, NC, USA.

凶e-mail:keisha_gibson@med.unc.edu

https://doi.org/10.1038/s41581-021-00520-5

1. Bignall, O. N. R. II \& Crews, D. C. Stony the road we trod: towards racial justice in kidney care. Nat. Rev. Nephrol. 17, 79-80 (2021).

2. Nguyen, K. H. et al. Evaluation of racial, ethnic, and socioeconomic disparities in initiation of kidney failure treatment during the first 4 months of the COVID-19 pandemic. JAMA Netw. Open 4, e2127369 (2021).

3. Eberly, L. A. et al. Association of race/ethnicity, gender, and socioeconomic status with sodium-glucose cotransporter 2 inhibitor use among patients with diabetes in the US. JAMA Netw. Open 4, e216139 (2021).

4. Golestaneh, L. et al. The role of place in disparities affecting Black men receiving hemodialysis. Kidney Int Rep. 6, 357-365 (2020).

5. Ahmed, S. et al. Examining the potential impact of race multiplier utilization in estimated glomerular filtration rate calculation on African-American care outcomes. J. Gen. Intern. Med. 36, 464-471 (2021).

6. Zelnick, L. R., Leca, N., Young, B. \& Bansal, N. Association of the estimated glomerular filtration rate with vs without a coefficient for race with time to eligibility for kidney transplant. JAMA Netw. Open $\mathbf{4}$, e2034004 (2021).

7. Delgado, C. et al. A unifying approach for GFR estimation: recommendations of the NKF-ASN Task Force on reassessing the inclusion of race in diagnosing kidney disease. Am. J. Kidney Dis. https://doi.org/10.1053/j.ajkd.2021.08.003 (2021).

8. Purnell, T. S., Simpson, D. C., Callender, C. O $\&$ Boulware, L. E. Dismantling structural racism as a root cause of racial disparities in COVID-19 and transplantation. Am. J. Transplant. 21, 2327-2332 (2021).

9. Danziger, J., Mukamal, K. J. \& Weinhandl, E. Associations of community water lead concentrations with haemoglobin concentrations and erythropoietinstimulating agent use among patients with advanced CKD. J. Am. Soc. Nephrol. 32, 2425-2434 (2021).

\section{Competing interests}

The authors declare no competing interests. 